\title{
A Design of ZigBee-based Intelligent Home Monitoring System
}

\author{
Lishuang Zhao \\ College of information science and Technology, Bohai University, Jinzhou, P.R. China \\ jz_zls@163.com
}

Keywords: Evaluation wireless sensor network; ZigBee; intelligent home; environment monitoring

\begin{abstract}
The thesis deals with the design of intelligent household environment monitoring equipment by the use of ZigBee technology. The system design takes STC12c5a60s2 single-chip microcomputer as the core module of data acquisition terminal. It is a ZigBee-based wireless sensor network acquisition system with low complexity, low power consumption and low cost. The sensor data display capabilities are realized through the single-chip microcomputer. The design mainly consists of the ZigBee wireless sensor network, a single-chip computer and a display device, with the single-chip computer as its core controller. The ZigBee chip CC2530 builds a wireless network for the core, carries temperature/humidity sensors, smoke sensor and infrared sensing devices, which constitute a complete environmental data acquisition system.
\end{abstract}

\section{Introduction}

With the constant development of wireless telecommunication technology, wireless sensor network has expanded from the earlier military field to industry, science and medicine and is playing an increasingly important role [1]. Wireless sensor network data acquisition technology has evolved from simplification to integration and networking. The ZigBee is a wireless network technology which is a low rate personal area. It's suitable for such occasions which not amount of data communication, relatively low data transmission speed, a high degree of data security and low power consumption [2]. The thesis deals with the indoor environment monitoring of intelligent households. The sensor network tests the data and the tested data are sent to the acquisition terminal wirelessly. The acquisition terminal analyzes data packet, extracts valid data and displays them on LED 12864.

\section{System Design Layout and Device Choice}

\section{System Framework Layout}

The system mainly consists of acquisition terminal and sensor network. The whole structure is illustrated in Fig. 1. The acquisition terminal uses a core controller STC12c5a60s2 single-chip computer which is 8-Bit, low-power-consumption and manufactured by domestic corporations. The computer communicates with the coordinator through the serial port. The coordinator ZigBee receives data sent from wireless acquisition node. The core controller analyzes the collected data, extracts valid data and displays them on LCD12864. The sensor network consists of ZigBee node merging sensors.

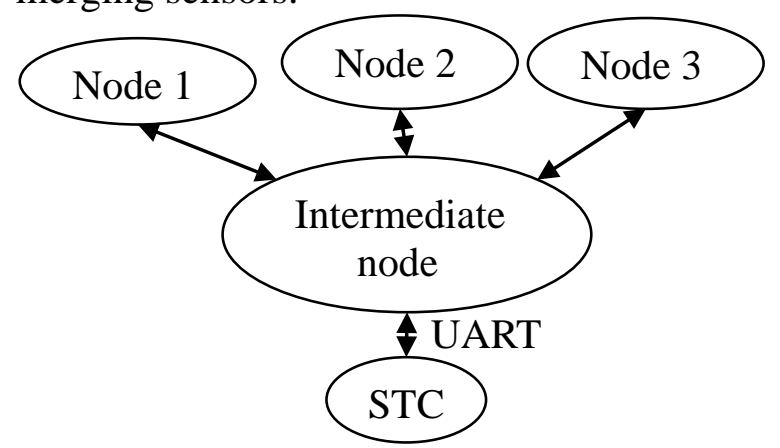

Fig. 1. The system structure frame

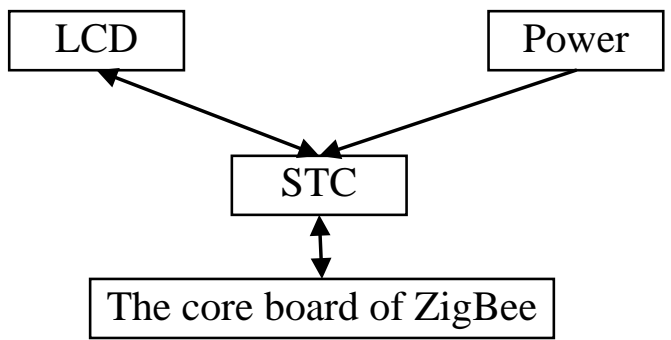

Fig. 2. Block diagram of control terminal for data acquisition system 


\section{Sensor Network Hardware Design}

The sensor network is designed to realize data acquisition of ZigBee device group network and indoor environment. It is composed of ZigBee node, power module and sensor acquisition module.

ZigBee subnet consists of nodes and sensors. The main control chip of nodes uses CC2530 single-chip computer by TI Company. The chip takes the design of low voltage and low power consumption. The internal features 256KB Flash, an 8-channel 12-Bit AD converter and a watchdog timer. Connected to CC2530 single-chip computer pin, the sensor sends the collected data message to CC2530 computer which further processes the data [3].

Wireless acquisition node is powered by $4.8 \mathrm{~V}$ lithium battery. The $3.3 \mathrm{~V}$ power supply of CC2530 computer is completed by dc level conversion through am1117 chip.

(1)Temperature/humidity sensor takes DHT11 digital temperature/humidity sensor, whose digital signal output has been calibrated. The product is of high reliability and excellent long-term stability.

(2)Gas leakage alarm MQ-2 is long-lived and low-cost. Simple drive circuit is highly sensitive to liquid gas, propane and hydrogen. When there are combustible gases in air, the electrical conductivity of the sensor increases with the increasing level of the combustible gases in air, and sends out digital signals.

(3)HC-SR501 is an auto control module based on infrared technology. Its original probe LHI778 is imported from Germany, with high sensitivity, reliability and ultra-low-voltage working model. It is widespread used in various auto inductive electrical appliances, especially in the auto control products of dry battery power supply.

The data interface of the three sensors takes the unibus structure, so data pin is directly connected to CC2530 single-chip computer P0.4.

3. Acquisition Terminal Hardware Design

Illustrated in Fig. 2, the core of acquisition terminal is the minimum system of STC12c5a60s2 single-chip computer. Data acquisition is completed through serial ports communication by connecting PO_2, PO_3 terminals (serial circuit) of CC2530 single chip to the terminal of STC12c5a60s2 single chip. Valid data is transmitted through P1 port and displayed on LCD12864. Acquisition terminal power is supplied by alternating current $220 \mathrm{~V}$, which is converted into $5 \mathrm{~V}$ and 3.3V and supplies direct current for acquisition terminal [4].

\section{System Software Design Layout}

\section{Acquisition Terminal Software Design}

The design of acquisition terminal software is comprised of coordinator and acquisition terminal. Illustrated in Fig. 3, the coordinator fulfills wireless data transmission and networking. The wireless communication can be realized by the coordinator. The sensor network data is firstly transmitted to the coordinator which then sends data to the acquisition terminal through serial ports and displays the operation and so on. At the same time, the coordinator also serves as the data sender, the coordinator can also transmit orders of acquisition terminal to wireless sensor, thus completing data intercommunication.

The core STC single chip of acquisition terminal sends out orders to wireless sensor for data collection [5]. The collected data are transmitted to STC single chip for display. The procedure flowchart of acquisition terminal is illustrated in Fig. 4.

The design of communication format completes data packet transmission by data frame formats. The data frame formats consist of frame header, address, data frames, command frame and frame end. Frame head and end are fixed bytes 9AH [6]. The address consists of two bytes. The control code consists of single byte. Data length is decided by data code. The data transmission format is illustrated in Table 1.

Table 1. RREP

\begin{tabular}{|c|c|c|c|c|c|c|}
\hline \multicolumn{8}{|c|}{ RREP } \\
\hline Frame Header & \multicolumn{2}{|c|}{ Address } & Control Code & DLC & Data Code & Frame End \\
\hline 9AH & A0 & A1 & C & L & DATA & 9AH \\
\hline
\end{tabular}




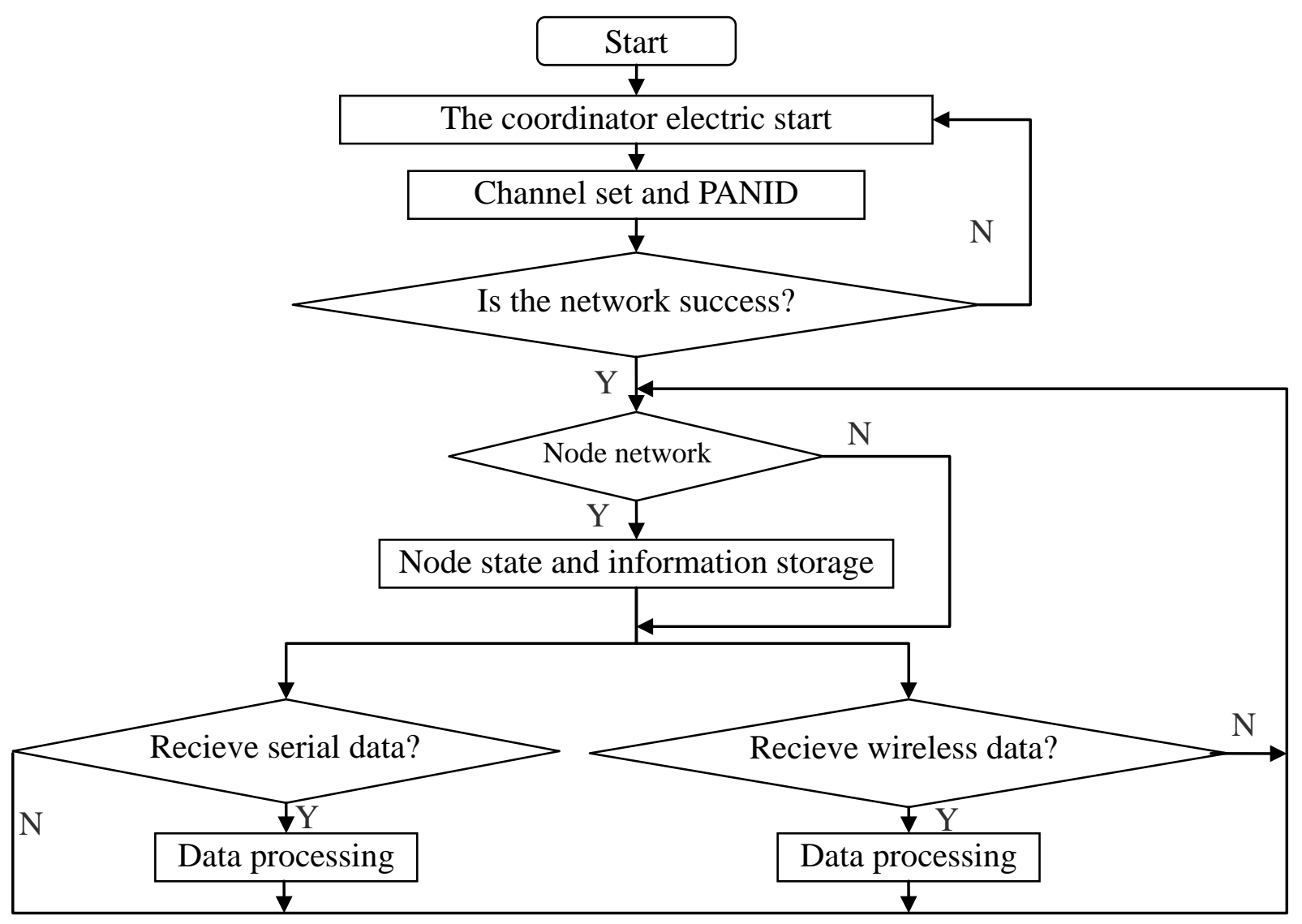

Fig. 3. The program flow diagram of coordinator

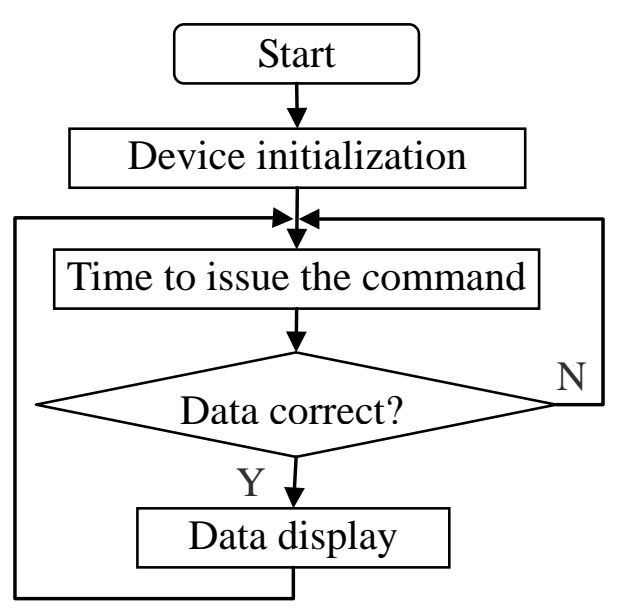

Fig. 4. The acquisition terminal flow chart

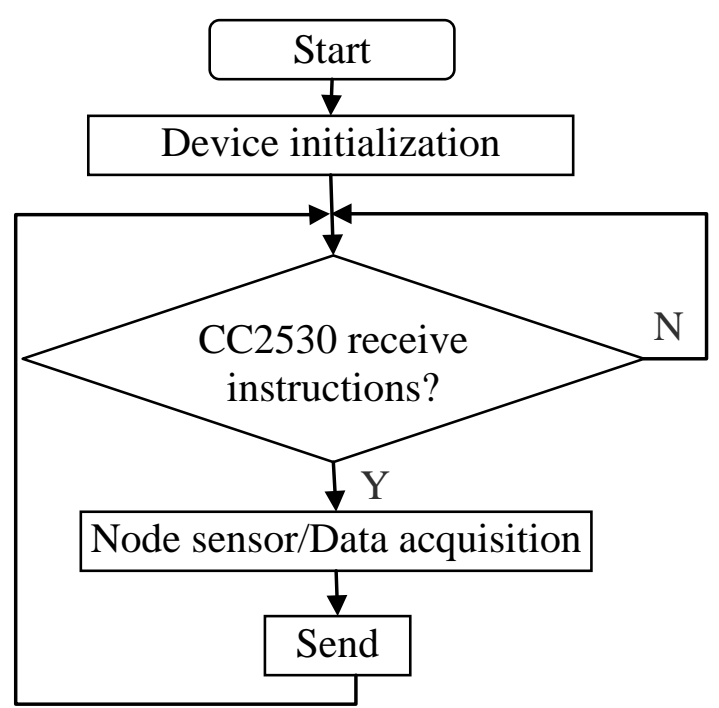

Fig. 5. Measuring node program flow chart

\section{Data Acquisition Node Software Design}

The design of data acquisition node software includes ZigBee node networking, processing the received instructions, operating in accordance with the instructions and auto starting work design. Taking the short distance into account, data acquisition nodes are all terminal nodes. The device is automatically networked after being started [7]. Then network information of the node is sent to the coordinator and acquisition terminal through network. Nodes in carries on corresponding processing received from the coordinator of instruction. It is illustrated in Fig. 5. 


\section{System Testing}

In order to realize the function of the system, we'll need for comprehensive testing of the system to understand the performance of the system. Because this paper adopts the ZigBee wireless network technology to the data transmission and each monitoring point management, So the main testing of this system is testing data transceiver and network performance by using the ZigBee wireless network technology.

The wireless network consists of one coordinator and four terminal nodes. The terminal nodes are temperature/humidity sensor, smoke sensor and the infrared induction device [8]. When a node and the measurement node distance is long, there will be a certain degree of signal attenuation. So we will need to test the communication distance of wireless. The transformation range of the signal intensity which read from the RSSI register of CC2530 is from 0 to 255 . This test is in 30m between the inner within a distance of two node test, the result of the test is very good.

Test 1, when each ZigBee node is added to the network, the network topology can show the correct.

Test 2, the ZigBee node on the transmission of data over can correctly displayed from the acquisition and control terminal.

Through the result of wireless network performance can be seen that the design which is based on wireless network sensors characterized by stronger network reliability, network expanding ability and data transmission stability. It can adapt to complex household environment monitoring.

\section{Conclusion}

Along with the people to the safe, comfortable, convenient living environment demand, a kind of intelligent home furnishing monitoring system based on ZigBee wireless network technology is designed. The design takes STC12c5a60s2 as the core module of data acquisition terminal. It's a ZigBee-based wireless sensor system with low complexity, low power consumption and low cost. It can adapt to the complex Home Furnishing environment by mounting temperature humidity sensor, smoke sensor and the infrared induction device. Compared with the traditional methods, this system has many merits, such as easy maintenance, high precision, good expansibility and flexibility. It can be easily used in the practical applications.

\section{References}

[1] Wan Zhiping, JinYongmin, Yang Yihong, "Design of a wireless data acquisition system based on ZigBee," Information Technology, vol. 2, no. 9, pp. 22-23, 2009.

[2] Dai Qi, "Disign of Wireless Streetlight Control System Based on ZigBee," Master's degree of Xiadan University, 2012.

[3] Wang Jianguo, Yang Jing, "Research for wireless sensor networks quality of service on the ZigBee protocol, " Electronic Design Engineering, vol. 22, no. 20, pp. 138-140, 2012.

[4] Wang Hao, "Design and Implementation of Temperature and Humidity Gathering Communication System Based on ZigBee Technology," Journal of Yancheng Institute of Technology(Natural Science Edition), vol. 26, no. 1, pp. 64-68, 2013.

[5] Hao Zhihua, Zheng Huanxia, "Wireless Temperature and Humidity Measurement System Based on Zigbee and GPRS," Coal Mine Machinery, vol. 34, no. 4, pp. 254-257, 2011.

[6] Liu Zijing, Pei Wenjiang, "Research of Wireless Sensor Network Based on ZigBee Protocol," Computer Technology and Development ,vol. 19, no. 5, pp. 192-195, 2009.

[7] Liao Shuzheng, "Research,Implementation and Application of Network Layer for ZigBee Protocol Stack," Master’s degree of Sun Yat-Sen University, 2010. 
[8] Shi Pin, Zheng Ping, "Design of Wireless Data Acquisition System Based on ZigBee and PLC," Journal of Xihua University(Natural Science), vol. 29, no. 6, pp. 28-31, 2010. 